\title{
REAÇÕES DE ORTOMETALACÃO EM PIRIDINAS
}

Marcus .V. N. de Souza* e Mauro V. de Almeida

Departamento de Química, Universidade Federal de Juiz de Fora, 36036-330 Juiz de Fora - MG

Recebido em 29/11/00; aceito em 2/5/01

THE ORTHO METALATION REACTION IN PYRIDINES. In this paper we describe a powerful methodology for the regiospecific construction of polysubstituted aromatic and heteroaromatic compounds. The DoM reaction (direct ortho-metalation) comprises the deprotonation in position ortho of a aromatic or heteroaromatic containing DMG (directed metalation group) by strong bases, normally an alkyllithium reagent, leading to an ortho-lithiated species. These species, upon treatment with electrophilic reagents, gives 1,2 disubstituted products.

Keywords: ortho-metalation; directed metallation group; heteroaromatic compounds.

\section{INTRODUÇÃO}

Entre 1939-40, trabalhos independentes realizados por Gilman e Bebb $^{1}$ e Wittig e Fuhrman ${ }^{2}$ demonstraram a ortodesprotonação do anisol em presença do $n$-butillítio, o qual se mostrou um método eficaz de funcionalização regiosseletiva de compostos aromáticos ${ }^{3 a, b}$. Este método foi estudado e posteriormente aplicado com sucesso em sistemas heteroaromáticos $\pi$ - deficientes (piridinas, quinolinas e diazinas) $)^{4}$.

O princípio da reação de metalação ortodirigida (DoM - "direct ortho-metallation"), em sistemas aromáticos baseia-se na desprotonação orientada em posição orto por um grupo diretor de metalação adequado (DMG - "direct metallation group"). A espécie ortolítio formada reage, por sua vez, com o eletrófilo $\left(\mathrm{E}^{+}\right)$desejado para formação de produtos 1,2-dissubstituídos (Figura 1).

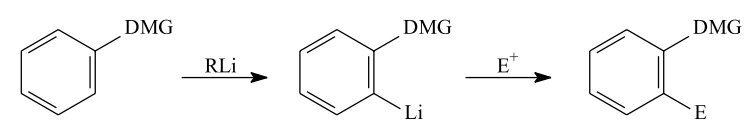

Figura 1. Princípio da reação de metalação ortodirigida

Este tipo de reação foi aplicado com sucesso em escala industrial por diferentes indústrias químicas, como por exemplo na prepara- ção de cardiotônicos do tipo $\mathbf{1}^{5}$ ou, mais recentemente, no desenvolvimento de análogos da sacarina $\mathbf{2}^{6}$ (Esquema 1$)$.

A reação de metalação ortodirigida depende principalmente de dois parâmetros:

1) Da natureza dos agentes de metalação: as bases fortes podem ser classificadas em duas grandes categorias ${ }^{7}$ :

* Os alquil e aril lítios (metillítio, $n$, sec, tert-butillítio, fenillítio, etc.) quelados ou não por ligantes, tais como $N, N, N^{\prime}, N^{\prime}-$ tetrametiletilenodiamina (TMEDA), que têm por finalidade reduzir o estado de desagregação da base ${ }^{8}$.

* Os alquilamidetos de lítio tais como, $N, N$-diisopropilamideto de lítio (LDA) e o hexametildisililamideto de lítio (LHMDS).

2) Da natureza do grupo diretor de metalação (DMG).

Os DMGs têm uma dupla influência racionalizada em termos da CIPE (Complexe-Induced Proximity Effects) ${ }^{9}$, sobre a primeira etapa crucial de desprotonação (Figura 2).

* Seu efeito indutivo aumenta a acidez do hidrogênio situado em orto.

* Eles interagem com a base litiada dirigindo-a ao sítio de desprotonação, permitindo assim a formação de uma espécie orto-litiada estabilizada por quelação.

É importante ressaltar também que o DMG deve possuir um fraco caráter eletrófilo em relação às bases litiadas utilizadas. Os DMGs<smiles>C#C[C@@H](C(=O)Nc1ccc(O)c(O)c1C(=O)O)C(=O)Nc1ccc(OC(C)C)c(C(=O)NCC)c1C(C)C</smiles>

Esquema 1. Reação de ortometalação aplicada em escala industrial na preparação de cardiotônicos do tipo 1 ou no desenvolvimento de análogos da sacarina 2.

*e-mail: mvndesouza@bol.com.br 

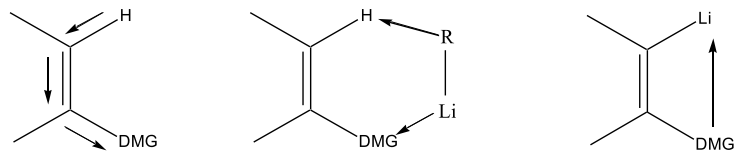

Figura 2. A reação de metalação ortodirigida depende principalmente da natureza dos agentes de metalação e do grupo diretor de metalação (DMG).

mais utilizados para reações de ortometalação dirigidas em sistemas piridínicos pertencem aos seguintes grupos:

\author{
Halogênios \\ NHCOR e $\mathrm{NHCO}_{2} \mathrm{R}$ \\ $\mathrm{OR}$ e OCONR \\ 2-oxazolino, CONHR, $\mathrm{CONR}_{2}$ \\ $\mathrm{SO}_{2} \mathrm{NR}_{2}$
}

\section{ORTOMETALAÇÃO DE HALOGENOPIRIDINAS}

Na década de 60 , Chambers $^{10}$, seguido por Abramovich ${ }^{11}$, foram os primeiros a realizarem reações de ortometalação sobre piridinas polifluoradas (Esquema 2). Nos anos 70, Quéguiner, estudando trocas halogênio-metal em bromopiridinas, descobriu que o bromo poderia funcionar como um grupo ortodiretor ${ }^{12}$. Esta observação foi em seguida confirmada pelas reações de ortometalação de outros compostos halogenados ou alcoxi-piridinas realizadas por Quéguiner $^{13}$, Suschitzky ${ }^{14}$, Wakefield ${ }^{15}$ e Gribble ${ }^{16,17}$.

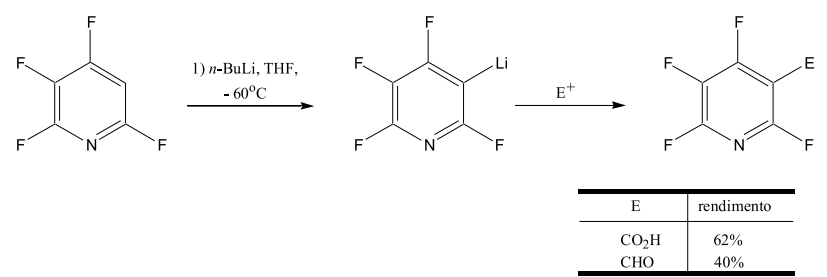

Esquema 2. Reações de ortometalação sobre piridinas polifluoradas realizadas por Chambers e Abramovich.

As reações de ortometalação dirigidas sobre compostos heterocíclicos aromáticos são de grande interesse por duas razões:

1) Os derivados halogenados são facilmente preparados a partir de uma função amina, hidroxila ou a partir de sistemas insaturados.

2) O halogênio pode ser utilizado para introduzir funcionalidade graças a reações de troca metal-halogênio, de substituição nucleofílica aromática $\left(\mathrm{S}_{\mathrm{N}} \mathrm{Ar}\right)$ ou de acoplamento (Esquema 3).

Além das vantagens acima mencionadas, muitas destas reações são mais fáceis de serem realizadas sobre compostos heterocíclicos aromáticos que sobre aqueles puramente aromáticos. Devido a este fato, a DoM permite o pronto acesso a diferentes produtos heterocíclicos aromáticos substituídos, dificilmente sintetizáveis por métodos clássicos.

\section{Quimiosseletividade}

A reação de um alquillítio com derivados halogenados da piridina, quinolina ou diazina pode dar origem a reações secundárias que competem consideravelmente, algumas vezes, com a reação esperada. Por exemplo, reações de substituição nucleofílica (via um mecanismo de adição-eliminação ou por um intermediário do tipo arino), abertura do anel heteroaromático, migração de halogênio e finalmente reações de acoplamento.

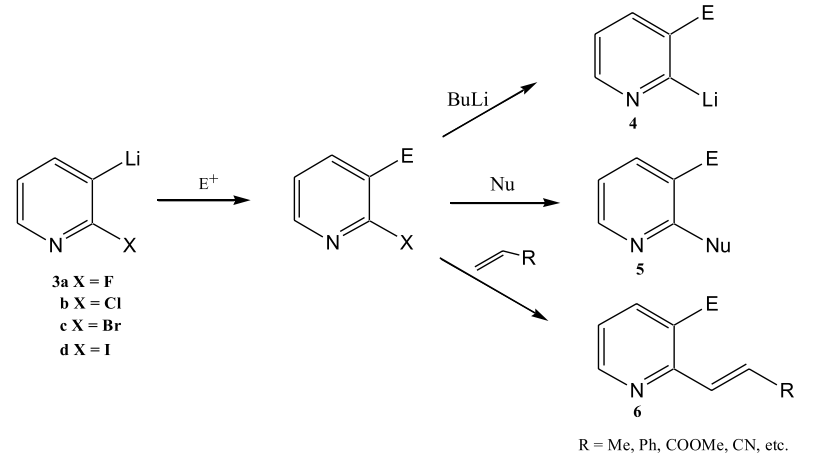

Esquema 3. Versatilidade das reações de ortometalação dirigidas sobre piridinas halogenadas.

\section{Adição Competitiva}

A utilização da 2-fluorpiridina 7a, 2-cloropiridina $7 \mathbf{b}$ ou da 2bromopiridina 7c, em presença de $n$-butillítio e de TMEDA em éter a $-40{ }^{\circ} \mathrm{C}$, conduz ao produto de adição nucleofílica em posição C-6 8. Após a adição do eletrófilo, os únicos produtos isolados foram as correspondentes 2,5-dihidropiridinas 9 (Esquema 4$)^{18}$.

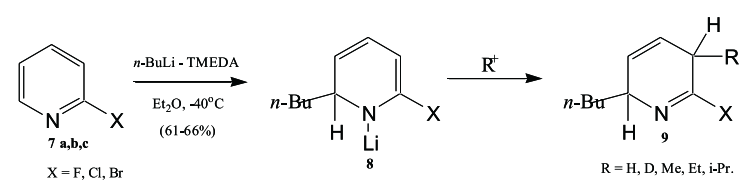

Esquema 4. Adição competitiva em reações de ortometalação de 2halogenopiridinas.

Quando a reação de metalação da 2-fluorpiridina 7a foi realizada a baixa temperatura $\left(-75^{\circ} \mathrm{C}\right)$ a formação dos produtos 10 e 11 (Esquema 5) foi constatada pela adição de cloreto de trimetilsilila (TMSCl). Uma quimiosseletividade completa da reação pode ser observada graças à utilização de uma base mais seletiva: o LDA a $75^{\circ} \mathrm{C}^{18,19}$.

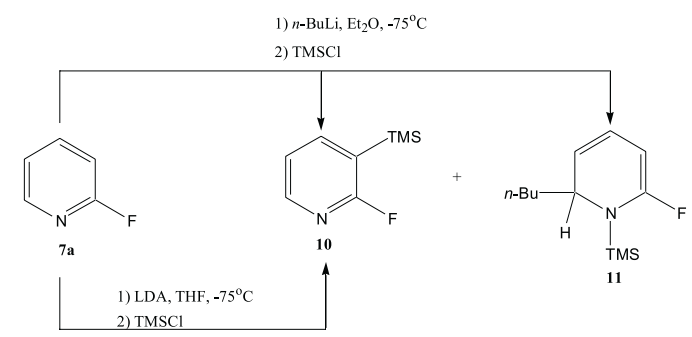

Esquema 5. Quimiosseletividade na reação de ortometalação da 2 fluorpiridina

\section{Formação Competitiva de Arinos}

Kauffman demonstrou que halogenopiridinas, em presença de um excesso de dialquilamideto de lítio a temperatura ambiente, pode provocar a formação de um intermediário do tipo arino 12. Uma adição nucleofílica, consequentemente, produz os compostos 13 e 14 (Esquema 6) ${ }^{20}$. Este tipo de reação de eliminação pode ser evitada diminuindo-se a temperatura da mesma.

A reação de metalação da 2-bromopiridina 7c, em presença de 1 equivalente de $\mathrm{LDA}$ a $-75^{\circ} \mathrm{C}$, permitiu a formação do intermediário litiado que pode ser evidenciado pela adição de $\mathrm{TMSCl}$, conduzindo 


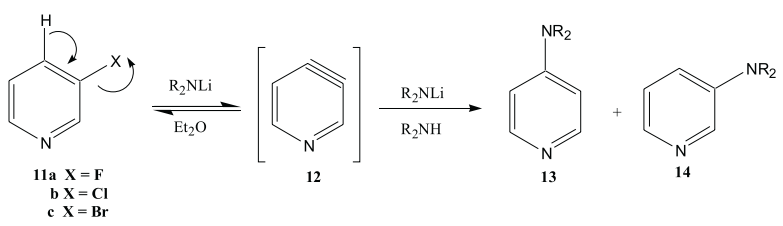

Esquema 6. Reações competitivas de arinos em reações de ortometalação de piridinas halogenadas.

assim ao produto 15. Quando quatro equivalentes de LDA são utilizados, ocorre a formação do produto 17, que pode ser explicada pelo ataque nucleofílico do LDA sobre a posição C-6 do anel piridínico (Esquema 7).
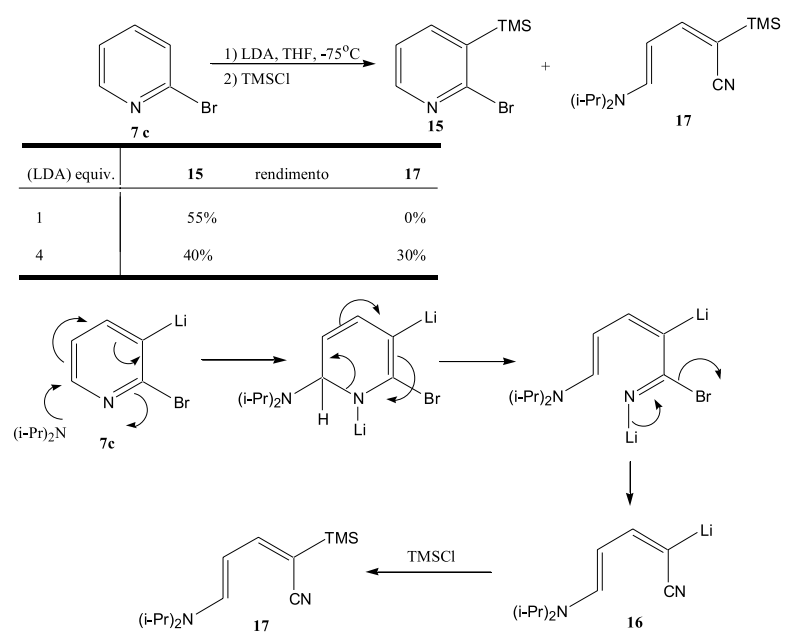

Esquema 7. Reação competitiva de abertura do anel piridínico em ortometalação de piridinas halogenadas.

\section{Migração Competitiva dos Halogênios}

Em 1972, Quéguiner e colaboradores descobriram a formação da 4-bromopiridina, após reação da 3-bromopiridina com excesso de $n$-butillítio a baixa temperatura ${ }^{12}$. O mecanismo proposto para este tipo de reação foi estudado utilizando-se as 3-bromopiridinas 18 (a,b,c) como suporte experimental ${ }^{22,23,24}$.

Esta reação de isomerização pode ser explicada por etapas sucessivas de trocas metal-halogênio e de homotransmetalação (Esquemas 8 e 9). Este último mecanismo foi proposto por Quéguiner para descrever a reação de ortometalação entre um material de partida e seu derivado litiado, resultante da reação com a base utilizada.

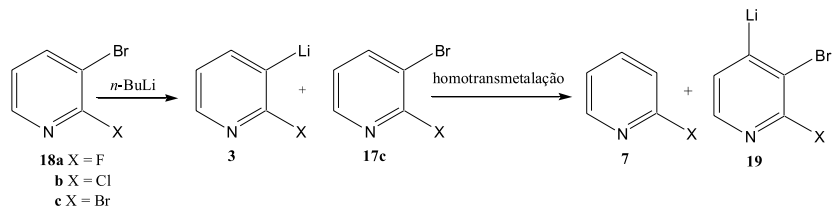

Esquema 8. Migração competitiva de halogênios em reações de ortometalação de piridinas halogenadas.

Os mecanismos destas transferências ("dança dos halogênios") 25-27, responsáveis pela isomerização, são suficientemente rápidos e reversíveis para que as espécies litiadas do tipo I e II estejam praticamente em equilíbrio (Esquema 10). Os parâmetros deste equilíbrio<smiles>[X]c1nccc(Br)c1Br</smiles><smiles>[Y]c1nccc(Br)c1Cl</smiles><smiles>[X]c1cc(Br)ccn1</smiles>

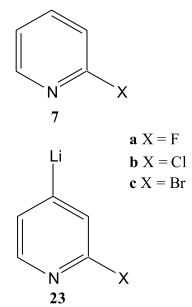

Esquema 9. Compostos presentes na reação de migração dos halogênios (dança dos halogênios).

(efeito indutivo destes halogênios, estabilização das espécies litiadas segundo a posição e estabilidade das ligações C-X), foram objetos de estudos teóricos detalhados ${ }^{18,23-25,28,29}$.

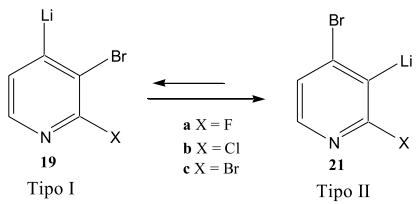

Esquema 10. Equilíbrio das espécies litiadas.

Concretamente, estas reações de isomerização foram observadas em outros compostos pela equipe de Quéguiner em reações de ortometalação, conduzidas em iodopiridinas com LDA a baixa temperatura (Esquema 11) ${ }^{30}$.
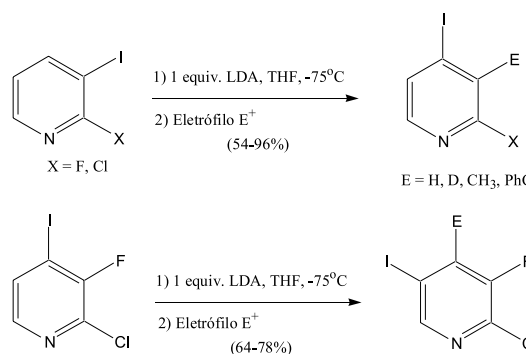

$\mathrm{E}=\mathrm{H}, \mathrm{D}, \mathrm{CH}_{3}, \mathrm{PhCH}(\mathrm{OH}), \mathrm{NH}_{2}, \mathrm{CHO}, \mathrm{I}$
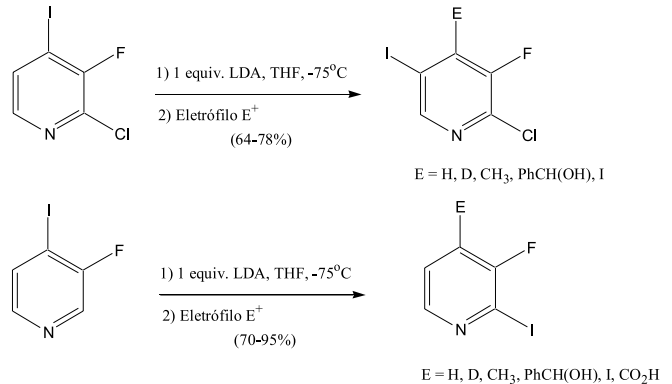

Esquema 11. Preparação de piridinas halogenadas substituídas via isomerização do iodo em reações de ortometalação.

\section{Reação de Acoplamento}

Em presença de LDA e HMPA (hexametilfosforamida) em solução etérea a $-70^{\circ} \mathrm{C}$, a piridina se dimeriza para formar a $2,2^{\prime}$-bipiridina 24 com um rendimento de $50 \%^{31}$. Resultados similares foram evidenciados com outras halogenopiridinas, como por exemplo a 3bromopiridina 11c, que nestas mesmas condições, porém a $-100^{\circ} \mathrm{C}$, produz a 3,3'dibromopiridina $\mathbf{2 5}^{32}$ ( Esquema 12).

\section{Regiosseletividade}

Estudos sistemáticos de regiosseletividade foram realizados em 3-cloropiridinas e 3-fluorpiridinas. Destacamos que a 3-fluorpiridina foi a primeira a ser utilizada em estudos de reações de metalação 


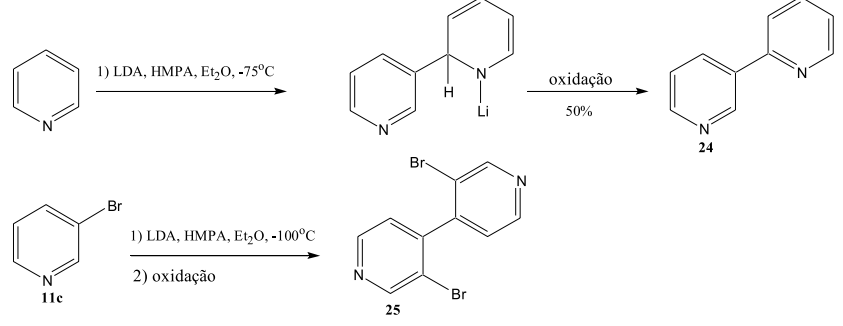

Esquema 12. Reações de dimerização em piridinas

ortodirigidas. Foi demonstrado que as condições reacionais utilizadas (solvente, temperatura, duração da reação e o agente de metalação) $)^{12}$ influenciam na regiosseletividade da reação de ortometalação.

\section{Influência do Solvente}

A ortolitiação da 3-fluorpiridina 11a em presença de $n$-BuLiTMEDA fornece os intermediários litiados 26 e 27. Depois da adição do TMSCl ou da 3-pentanona como eletrófilo os produtos $\mathbf{2 8}$, 29 e 30, 31 foram sintetizados, respectivamente. Os resultados indicados no Esquema 13 mostram que o intermediário litiado em posição 2 (26) predomina em éter e o intermediário litiado em posição 4 (27) em THF. A regiosseletividade da reação de ortometalação pode ser racionalizada em termos de controle cinético e termodinâmico (Esquema 13) ${ }^{33}$. O éter, sendo solvente menos básico que o THF, favorece a formação da espécie cinética 26, já que a coordenação do complexo $n$-BuLi-TMEDA com o nitrogênio da piridina se faz preferencialmente.

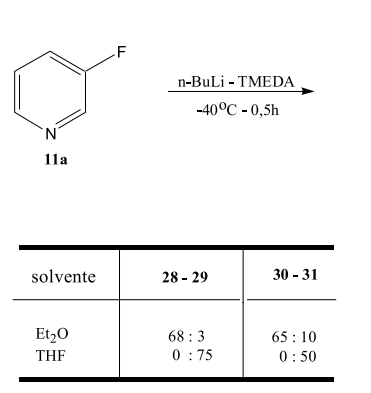

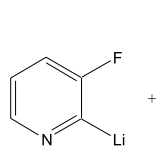

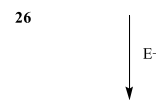

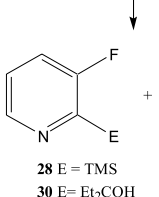

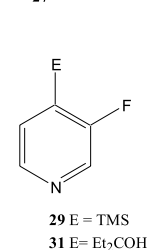

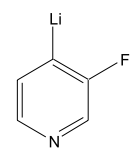

27

$31 \mathrm{E}=\mathrm{Et}_{2} \mathrm{COH}$

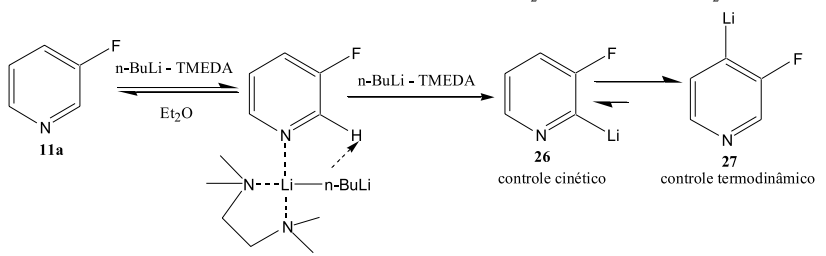

Esquema 13. Influência do solvente em reações de ortometalação de piridinas halogenadas.

\section{Influência da Temperatura e do Tempo de Reação}

A influência da temperatura e do tempo de reação foi igualmente estudada. A 3-fluoropiridina 11a foi submetida ao tratamento com o complexo $n$-BuLi-TMEDA a $-40^{\circ} \mathrm{C}$, adicionado pelo período de duas horas, para conduzir aos derivados litiados 26 e 27, cujas formações foram confirmadas pela adição de TMSCl como eletrófilo. Os resultados apresentados no Esquema 14 mostram que o equilíbrio entre as espécies litiadas se desloca com o tempo e o aumento da temperatura, do produto cinético $\mathbf{2 8}$ em direção ao produto termodinâmico $\mathbf{2 9}^{33}$.

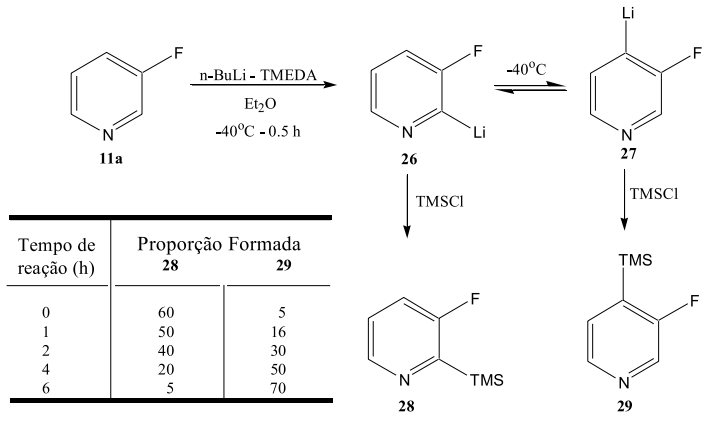

Esquema 14. Influência da temperatura e do tempo de reação em ortometalação de piridinas halogenadas.

\section{Agentes de Metalação}

Os amidetos de dialquillítios são bases com um fraco caráter nucleofílico e são mais eficazes que o $n$-BuLi-TMEDA a $0{ }^{\circ} \mathrm{C}$, condições clássicas para a ortometalação da 3 -cloropiridina $\mathbf{1 1 b}^{15,16,18}$. Entre os diferentes amidetos utilizados, o LDA se destaca como o melhor agente de metalação conduzindo, depois da adição de TMSCl, à piridina dissubstituída 33 com um rendimento de 96\% (Esquema 15).

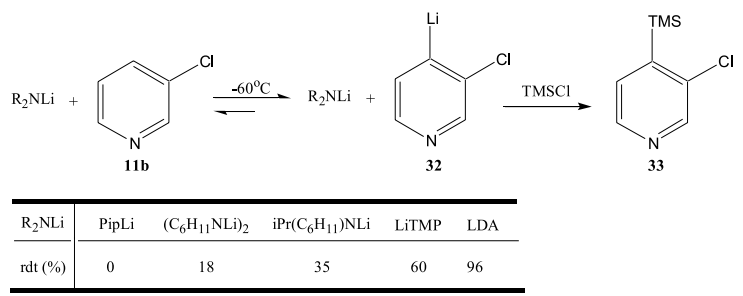

Esquema 15. Importância do agente de metalação em reações de ortometalação de piridinas halogenadas.

\section{Quantidade do Agente de Metalação}

As reações de ortometalação da 3-fluorpiridina 11a ou da 3cloropiridina 11b com LDA são muito sensíveis ao excesso de base (Esquema 16). A utilização de mais de um equivalente de LDA, seguido da adição de TMSCl como eletrófilo, produz os produtos 2,4substituídos 34 e $\mathbf{3 5}$, respectivamente. O rendimento da formação destes compostos é proporcional à quantidade de base adicionada em excesso ${ }^{33}$.

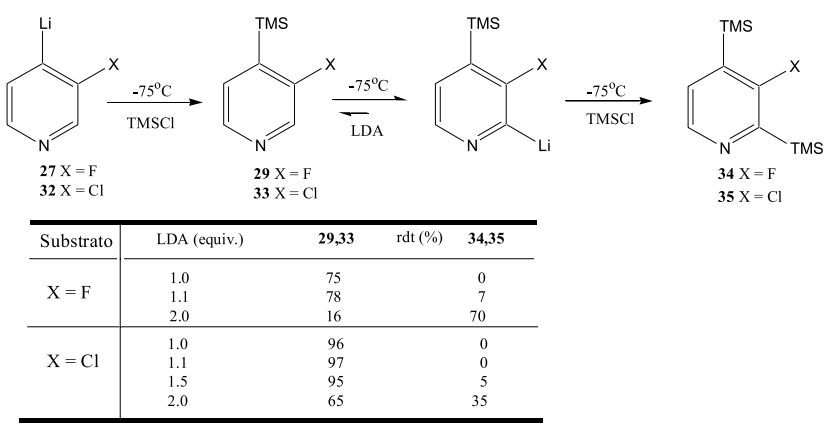

Esquema 16. Importância da quantidade do agente de metalação em reações de ortometalação de piridinas halogenadas. 


\section{Aplicações Sintéticas}

A ortometalação de heterociclos halogenados é uma reação que pode ser aplicada com eficácia no plano sintético. A título de exemplo apresentamos a síntese total da perlolidina $36^{3436}$ (Esquema 17), um alcalóide isolado de um tipo de gramínea da Nova Zelândia. A ortolitiação da 2 fluorpiridina pelo LDA, seguida da adição de iodo dá acesso ao produto iodado 37 com um rendimento de $80 \%$. A formilação da posição C-3 em uma só etapa, pela adição do formiato de etila, se faz concomitantemente com a migração do iodo da posição C-3 para a posição C-4, obtendo-se assim o intermediário 38 com $75 \%$ de rendimento.

Paralelamente, o ácido borônico $\mathbf{4 0}$ foi preparado em três etapas com um rendimento global de $58 \%{ }^{37}$ através de uma reação de ortolitiação de 39 com o $n$-butillítio em THF, a $-60{ }^{\circ} \mathrm{C}$, seguida da adição de $\mathrm{B}(\mathrm{OMe})_{3}$ e de uma hidrólise ácida. Os intermediários 38 e 40 foram então submetidos a uma reação de acoplamento do tipo Suzuki ${ }^{38}$ catalisada por compostos de paládio. A utilização do $\operatorname{Pd}\left(\mathrm{Ph}_{3}\right)_{4}$ e $\mathrm{K}_{2} \mathrm{CO}_{3}$, em uma mistura de etanol-tolueno em refluxo, permitiu efetuar simultaneamente a reação de acoplamento, a desproteção do grupo pivalamida e a ciclização da amina obtida com o aldeído, obtendo-se assim o produto 41 com um rendimento de $60 \%$. A hidrólise do derivado fluorado foi realizada pelo tratamento com uma solução $4,8 \mathrm{~mol} / \mathrm{L}$ de $\mathrm{HCl}$, sob aquecimento, permitindo a formação da Perlolidina 36 com um rendimento de $80 \%$.
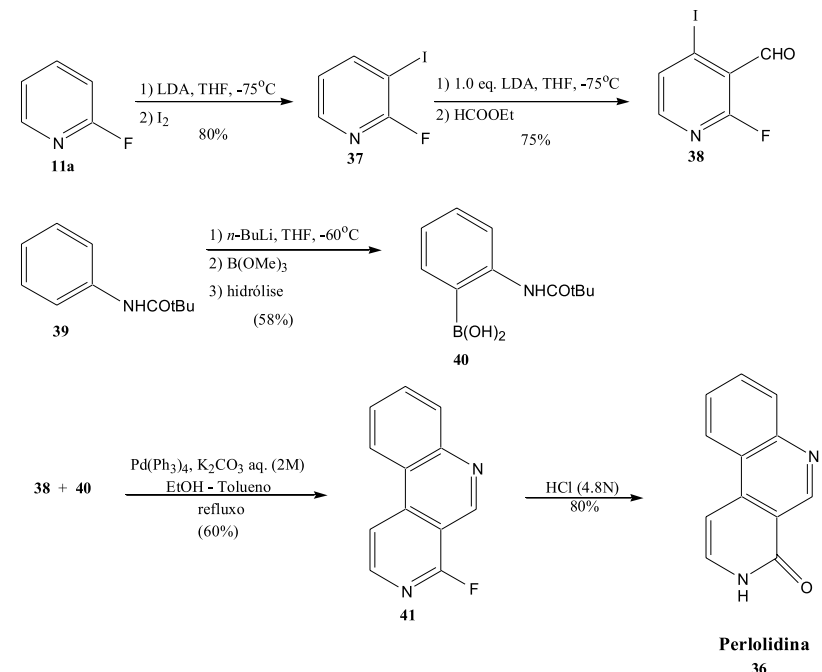

Esquema 17. Síntese da perlolidina 36

\section{ORTOMETALAÇÃO DE AMINOPIRIDINAS}

O estudo de metalação de aminopiridinas foi conduzido primeiramente sobre o composto 3-metoxi-5-pivaloilamino-piridina $\mathbf{4 2}^{39}$. Neste caso, o efeito combinado dos dois grupos ortodiretores proporcionou o fácil e seletivo acesso a compostos substituídos na posição C-4 usando-se $n$-butillítio e o respectivo eletrófilo (Esquema 18).

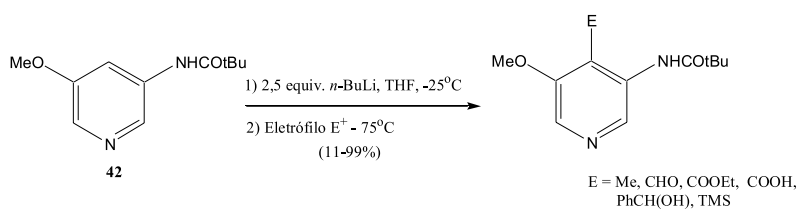

Esquema 18. Ortometalação da aminopiridina 42.

A combinação dos dois grupos ortodiretores (NHCOtBu e OMe) não é condição essencial para o sucesso da reação, como mostram os resultados do Esquema 19. Podemos ressaltar que a metalação da 2, da 3 ou da 4- $N$-pivaloilaminopiridina pela utilização de 2,5 equivalentes de $n$-butillítio, seguida da adição de diferentes eletrófilos, permite a obtenção das respectivas aminopiridinas ortossubstituídas com excelente regiosseletividade ${ }^{28,40,41}$ (Esquema 19).

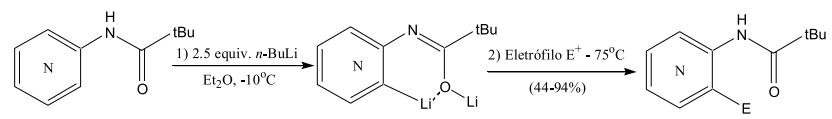

Esquema 19. Ortometalação de aminopiridinas.

Uma experiência realizada por Roland ${ }^{4}$ permitiu comparar o poder ortodiretor dos grupos Boc e pivaló́la. Os resultados obtidos demonstraram que o grupo $N$-pivaloíla possui um poder ortodiretor maior quando comparado ao $N$-Boc. Assim, a ortometalação de uma mistura equimolar dos compostos 43 e 44, seguida da adição de iodeto de metila, fornece seletivamente o produto de substituição em orto ao grupo pivalamida 45 (Esquema 20).
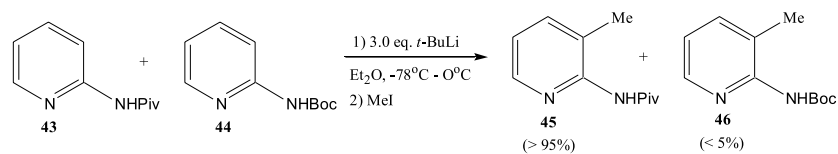

Esquema 20. Importância do grupo ortodiretor em ortometalação de aminopiridinas.

Além destes resultados mencionados anteriormente, a adição de 2,2 equivalentes de $t$-BuLi a uma solução de 4-metil-(2- $N$ pivaloil)aminopiridina 47 em éter, seguido da adição de iodeto de metila, fornece seletivamente o produto de ortometalação $\mathbf{4 8}$. Todavia, a aplicação destas condições reacionais ao derivado $N$-Boc 49 conduz essencialmente à formação do produto de alquilação do grupamento metila (composto 50, Esquema 21) .

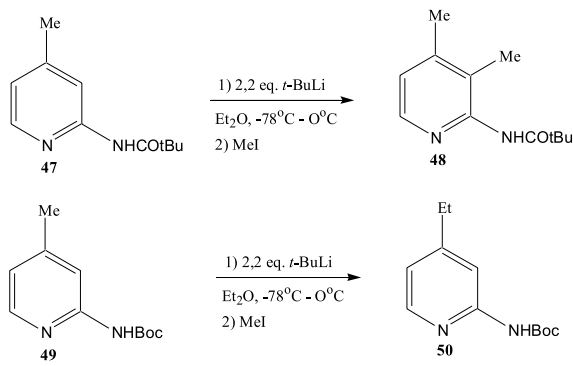

Esquema 21. Influência do grupo ortodiretor em reações de ortometalação versus alquilação em aminopiridinas.

\section{Aplicações Sintéticas}

A utilização do $N$-(pivaloil) aminopiridina como grupo ortodiretor permitiu, entre outras, a realização de uma eficiente síntese da tioazo[5,4-c]piridina 51 e da 3-tio-4-aminopiridina 52 (Esquema $22)^{42}$.

\section{ORTOMETALAÇÃO DE ALCOXI E CARBAMOILPIRIDINAS}

É também possível efetuar reações de ortometalação do anel piridínico utilizando uma cadeia oxigenada como grupo ortodiretor 

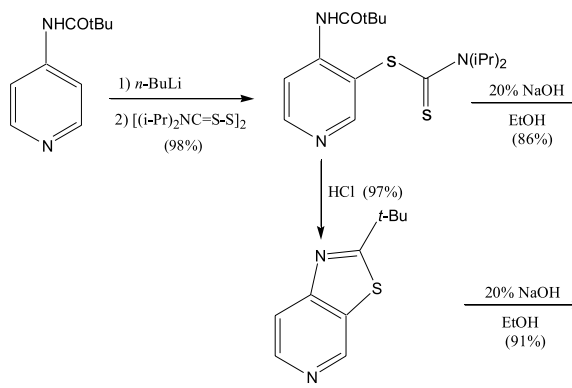

Esquema 22. Síntese da tioazo[5,4-c]piridina 51 e da 3-tio-4-aminopiridina 52 via reação de ortometalação de aminopiridinas.

principal. Os grupos mais freqüentemente utilizados são do tipo $\mathrm{OMe}^{7}, \mathrm{OMOM} \mathrm{OMEM}^{43,44}, \mathrm{OCOR}^{45}$ e OSEM $^{46}$.

\section{Ortometalação de Alcoxipiridinas}

$\mathrm{DMG}=$ Oalquila

Em 1982, Quéguiner e colaboradores demonstraram que a metalação de 3-alcoxipiridinas $\mathbf{5 3}$ poderia ser realizada seletivamente na posição C-2 em presença de $n$-BuLi-TMEDA (Esquema 23) ${ }^{28}$.

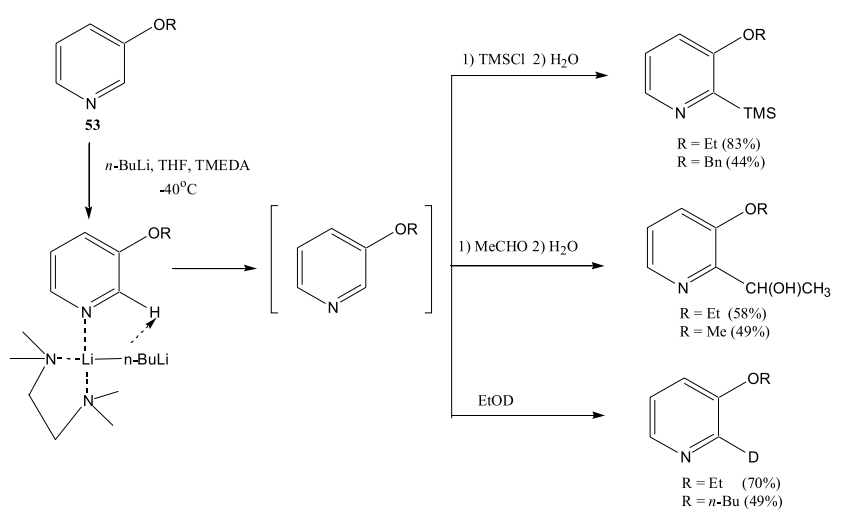

Esquema 23. Reações de ortometalação de alcoxipiridinas realizadas regiosseletivamente em posição $C-2$ em presença de $n$-BuLi-TMEDA.

$\mathrm{DMG}=$ Ometoximetila $(\mathrm{OMOM})-\mathrm{OCH}_{2} \mathrm{OCH}_{3}$

A iodação regiosseletiva da posição 4 do 3-O(MOM)-piridina 54 produziu o composto 55 com um rendimento de $90 \%$, utilizandose o $t$-BuLi como agente de metalação. Nestas condições somente traços do composto iodado em posição C-2 $\mathbf{5 6}$ foi isolado (Esquema $24)^{45,47}$. A inversão da regiosseletividade observada em relação a reação precedente foi explicada pelo estado de transição apresentado no Esquema 24, no qual a utilização de um agente de metalação volumoso, a baixa temperatura, conduz à desprotonação seletiva da posição C-4.

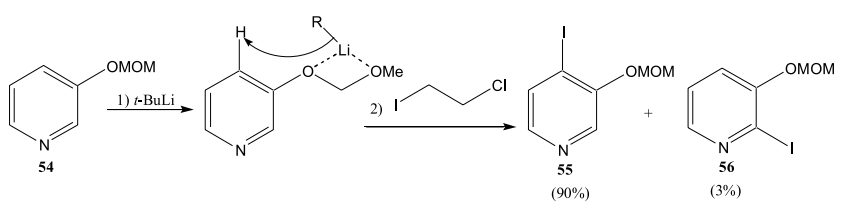

Esquema 24. Reações de ortometalação de alcoxipiridina realizada regiosseletivamente em posição $C$-4.
$\mathrm{DMG}=$ Oetoximetila $(\mathrm{OMEM})-\mathrm{OCH}_{2} \mathrm{OCH}_{2} \mathrm{CH}_{2} \mathrm{OCH}_{3}$

A utilização do $s$-BuLi como agente de metalação da 4-Oetoximetil-piridina 57, seguida da adição do TMSCl como eletrófilo, forneceu os produtos $\mathbf{5 8}$ e $\mathbf{5 9}$, devido à adição nucleofílica da base sobre o anel piridínico. Todavia, a utilização de 2,2 equivalentes de LDA conduziu à formação regiosseletiva de um único produto $\mathbf{6 0}$ com rendimento de $39 \%$ (Esquema 25$)^{48}$.

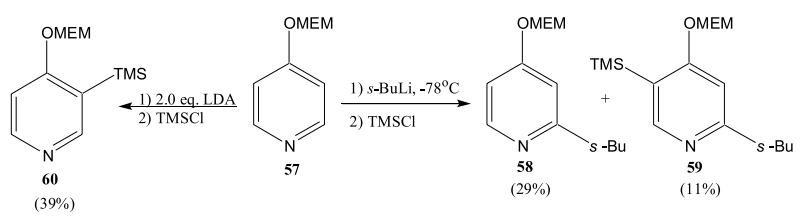

Esquema 25. Influência do agente de metalação em reações de ortometalação de alcoxipiridinas.

$\mathrm{DMG}=$ Otrimetilsililetoximetila $(\mathrm{OSEM})-\mathrm{OCH}_{2} \mathrm{OCH}_{2} \mathrm{CH}_{2} \mathrm{Si}\left(\mathrm{CH}_{3}\right)_{3}$

Snieckus e colaboradores ${ }^{49}$ demonstraram que a utilização do grupo 2-(trimetilsilil)-etoximetila (SEM), como grupo ortodiretor em posição C-3 de piridinas, produz excelentes resultados: os rendimentos das reações são elevados, os eletrófilos são introduzidos regiosseletivamente em posição $\mathrm{C}-4$ e os produtos formados possuem a vantagem de serem facilmente desprotegidos em condições brandas (Esquema 26)
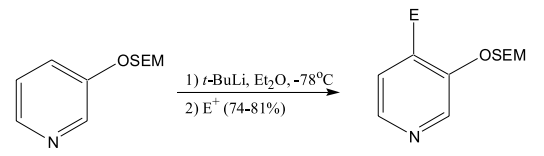

$\mathrm{E}=\mathrm{TMS}, \mathrm{Me}, \mathrm{PhCH}(\mathrm{OH})$

Esquema 26. Utilização do grupo SEM, 2-(trimetilsilil)-etoximetila como ortodiretor em reações de ortometalação em alcoxipiridinas.

\section{Ortometalação de Carbamoilpiridinas}

$\mathrm{O} N, N$-dietilcarbamato é um grupo ortodiretor muito útil para a síntese de hidroxipiridinas. Após o tratamento com $n$-BuLi-TMEDA a $-78^{\circ} \mathrm{C}$ em THF, é possível introduzir uma variedade de eletrófilos com rendimentos elevados ${ }^{50}$. A hidrólise do carbamato pode ser realizada em condições brandas (Esquema 27).

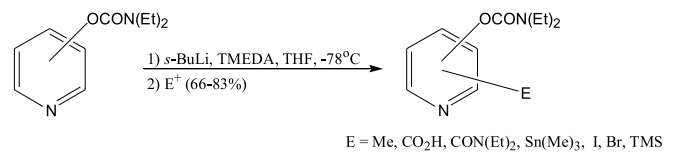

Esquema 27. Reações de ortometalação em carbamoilpiridinas

Além dos resultados apresentados anteriormente, os rearranjos iônicos do tipo orto-Fries, permitiram a transformação de $\mathbf{6 1}$ em 62 e 63 em 64, baseados em reações de ortometalação, igualmente possibilitando a preparação de hidroxipiridinas ortossubstituídas ${ }^{51}$ (Esquema 28).

\section{Aplicações Sintéticas}

A utilização de grupos ortodiretores oxigenados permitiu a síntese rápida e eficaz da azaxantona 65 e da 8 -azacumarina 66 $^{52}$ (Esquema 29). 


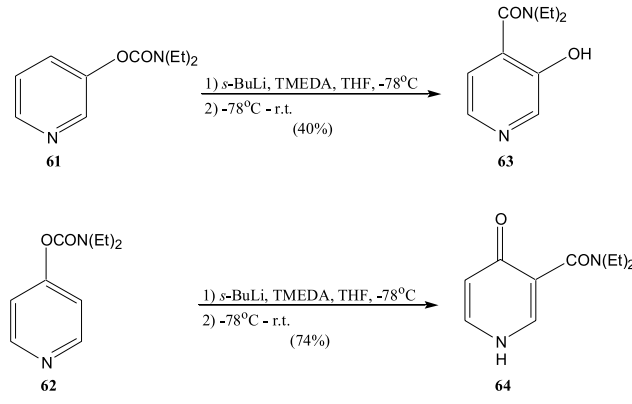

Esquema 28. Rearranjos iônicos do tipo orto-Fries, baseados em reações de ortometalação.

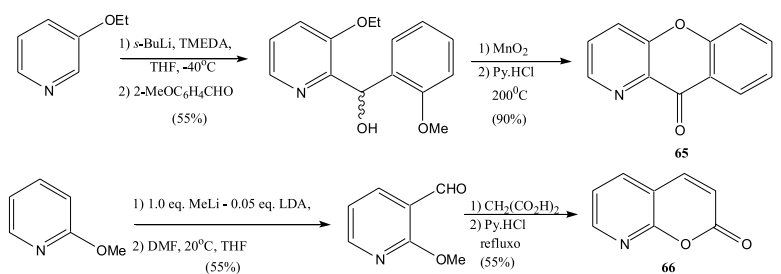

Esquema 29. Síntese da azaxantona 65 e da 8-azacumarina 66 via reações de ortometalação.

Uma outra ilustração da utilidade destes grupos ortodiretores foi apresentada pela primeira síntese realizada por Quéguiner e colaboradores $^{53}$ da caerulomicina C 67 (Esquema 30), um antibiótico isolado em $1959^{54}$ cuja estrutura foi somente elucidada em $1977^{55}$. A estratégia sintética envolve uma sucessão de reações de metalação, transmetalação, acoplamento aromático e migração de halogênio.
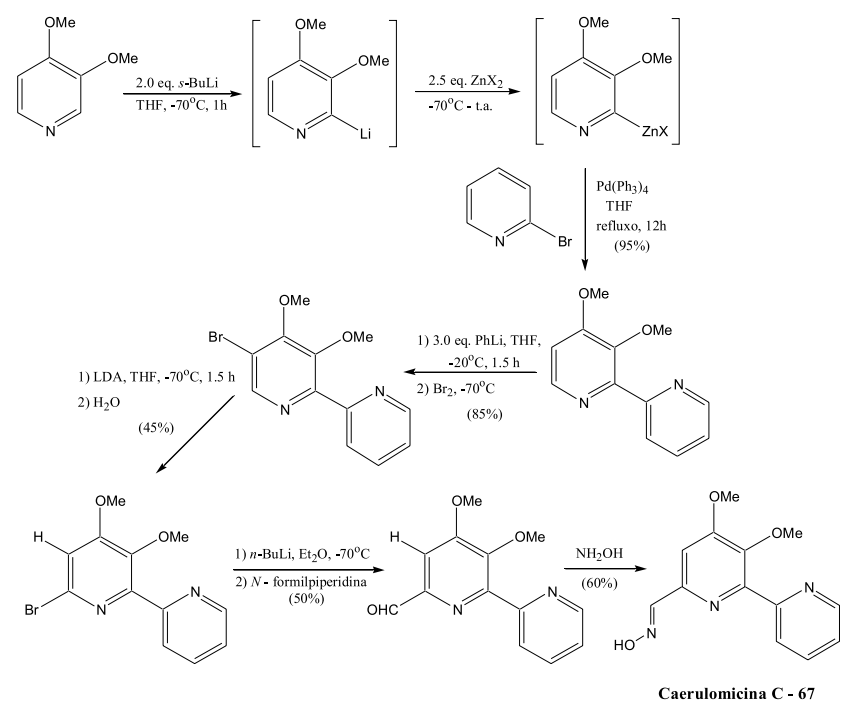

Esquema 30. Síntese da caerulomicina C 67.

\section{UTILIZAÇ̃̃̃O DE DERIVADOS DE ÁCIDOS CARBOXÍLICOS COMO GRUPOS ORTODIRETORES}

Em 1979, Ferles e Silhanka mostraram que o 4-nicotinoil nicotinato de etila 69 poderia ser obtido através do tratamento do nicotinato de etila 68 pelo LDA $^{56}$ (Esquema 31 ). A partir destes resultados a utilização de grupos ortodiretores carbonados foi desenvolvida por Snieckus e colaboradores ${ }^{57}$ e aplicada à química heterocíclica com bons resultados. A estratégia, baseada na utilização de 2-oxazolinas ${ }^{58}$, amidas secundárias ${ }^{59,60 a, b, c}$ e terciárias ${ }^{61,62}$ como grupos ortodiretores foi também aplicada ao anel piridínico.

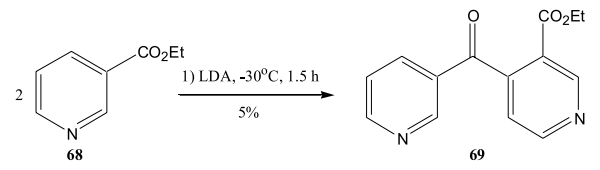

Esquema 31. Obtenção do 4-nicotinoil nicotinato de etila 69 utilizando-se LDA.

\section{Ortometalação de Oxazolinopiridinas}

A metalação da 4-oxazolinopiridina 70, utilizando-se $n$-BuLi ou $s$-BuLi, seguida da adição de iodeto de metila, produziu o composto 3-metilado 71 esperado acompanhado do produto de adição

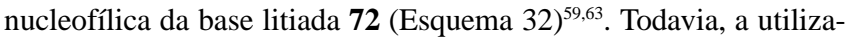
ção do metillítio como agente de metalação permitiu obter somente o produto de ortossubstituição $\mathbf{7 3}$, independente da natureza do eletrófilo. Estes resultados podem ser explicados devido ao menor tamanho do MeLi, o que facilita a coordenação com o grupo ortodiretor.

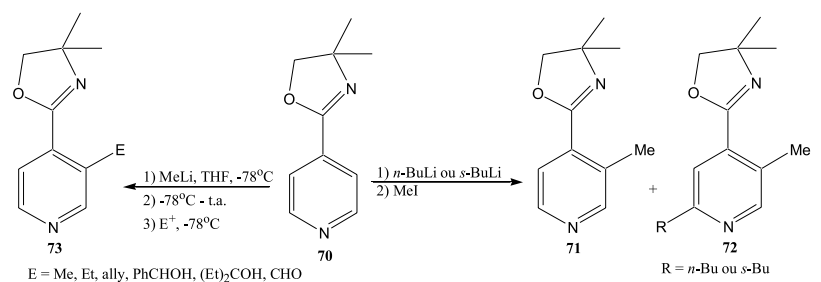

Esquema 32. Reações de ortometalação em oxazolinopiridinas.

\section{Ortometalação de Piridinas Carboxamidas Secundárias}

Experiências de metalação e de deuteração conduzidas sobre compostos contendo amidas secundárias como grupo ortodiretores, nas séries picolínica 74 e isonicotínica 75, mostraram que o rendimento da reação depende diretamente da natureza do substituinte ligado ao nitrogênio da amida secundária. Os resultados apresentados no Esquema 33 mostram que o melhor substituinte é o fenila ${ }^{64}$. A aplicação destas condições permite a preparação dos compostos ortossubstituídos correspondentes ${ }^{60}$.

\section{Ortometalação de Piridinas Carboxamidas Terciárias}

O estudo do uso da $\mathrm{N}, \mathrm{N}$-diisopropilamida como grupo ortodiretor na série de piridinas foi também realizado ${ }^{60}$ (Esquema 34). Entre outras, a litiação com LDA, seguida da adição de diferentes eletrófilos, permitiu o acesso aos produtos ortossubstituídos com rendimentos entre $25 \%$ a $75 \%{ }^{63,65}$.

\section{Aplicações Sintéticas}

Síntese da Ellipticina

A oxazolinopiridina $\mathbf{7 0}$ serviu de material de partida para a preparação do alcalóide anti-leucêmico ellipticina 76 (Esquema 35$)^{66}$. O intermediário-chave $\mathbf{7 7}$ foi preparado por uma reação de ortometalação de $\mathbf{7 0}$ com o MeLi. A adição de formaldeído, seguida de uma reação de ciclização em meio ácido, permitiu o acesso à 77 com um rendimento de $50 \%$. A lactona 77 formada foi condensada 


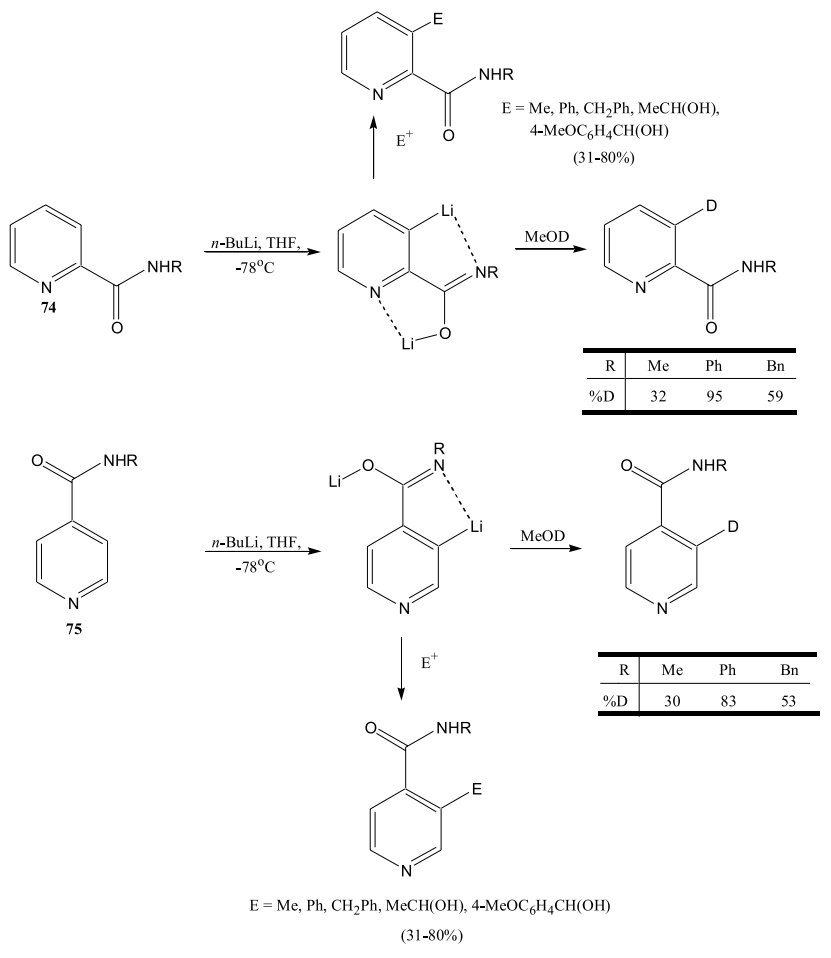

Esquema 33. Reações de ortometalação de carboxamidas secundárias.

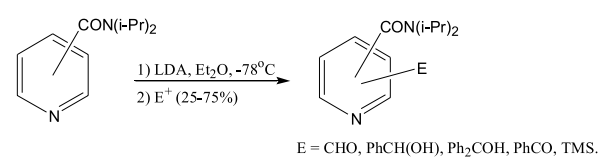

Esquema 34. Reações de ortometalação de piridinas carboxamidas terciárias. com o 2-indol-lítio 78, seguida da oxidação da função álcool, formando assim o aldeído 79, o qual foi transformado na quinona 80 e posteriormente na ellipticina $\mathbf{7 6 .}$

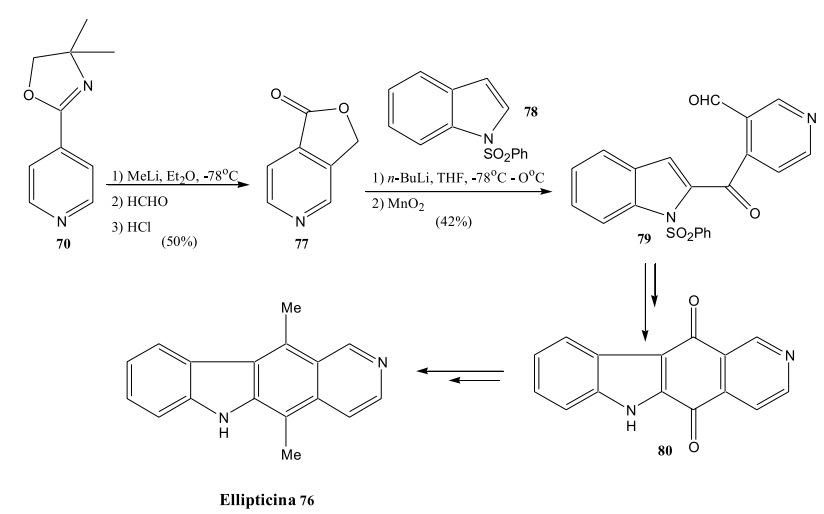

Esquema 35. Síntese da ellipticina 76 via reações de ortometalação.

Síntese do Ácido Berninamicínico

O ácido berninamicínico $\mathbf{8 1}^{67}$, produzido pela degradação da berninamicina, um antibiótico polipeptídico cíclico, foi sintetizado a partir da diamida 82 via uma reação de ortometalação. Estas condições permitiram, entre outras, a funcionalização da posição orto à amida secundária e a transformação da amida primária, obtendo-se o composto 83 com um rendimento de $67 \%$. Este intermediário, após cinco etapas reacionais, foi transformado no ácido berninamicínico (Esquema 36).

Síntese da Sesbanina

A utilização de amidas terciárias, como grupos ortodiretores, foi igualmente ilustrada na síntese da sesbanina 84 (Esquema 37), substância isolada em 1979 a partir de extratos etanólicos da sesbania<smiles>O=C(O)c1csc2c3ccc(C(=O)O)nc3c(O)c-2n1</smiles>

Ácido Berninamicínico 81

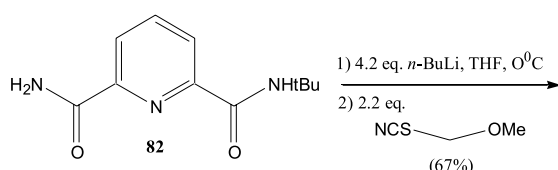<smiles>CCCCCCNC(=S)NC(=O)c1ccc(C(=O)NCOC)c(C(=O)NCCOC)n1</smiles><smiles>CCOC(=O)c1csc(NC(=O)c2ccc(-c3nc(C(=O)OC)cs3)c(C(=O)CN)n2)n1</smiles><smiles>CC(C)[Mg][Mg]</smiles><smiles>CCOC(=O)CBr</smiles><smiles>CCCCNC(=O)c1nc(C(=O)Nc2nc(C(=O)OCCOCCCC)cs2)ccc1-c1nc(C(=O)OCC)cs1</smiles>

Esquema 36. Síntese do ácido berninamicínico 81. 
dromondili $^{68}$ e que demonstrou uma potente atividade antileucêmica ${ }^{69}$. A etapa chave desta síntese é baseada numa reação de ortolitiação regiosseletiva da $N, N$-(diisopropil)nicotinamida, seguida de uma condensação com a 3-ciclopentenona, produzindo assim o intermediário spiro $\mathbf{8 5}^{70}$.

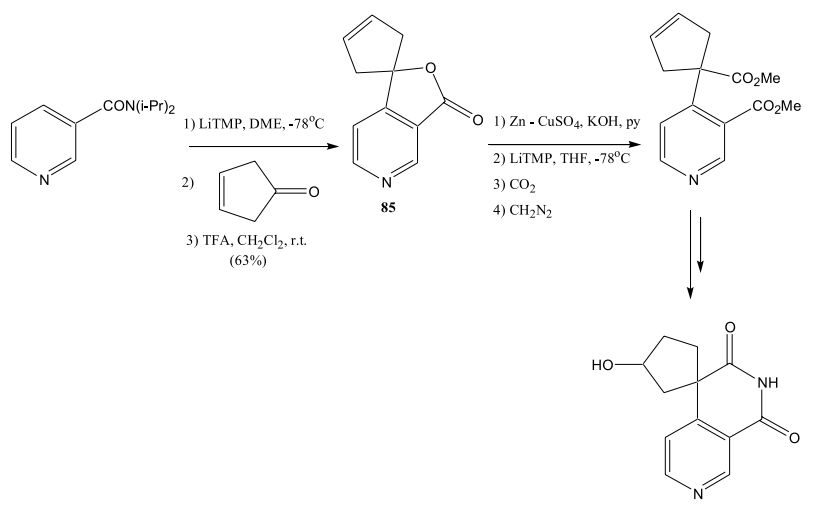

( \pm ) Sesbanina 84

Esquema 37. Síntese da sesbanina 84

\section{Utilização de Derivados de Enxofre como Grupos Ortodiretores}

A idéia de se utilizar grupos ortodiretores contendo enxofre foi concretizada pelo uso de grupos como os sulfetos $\mathrm{SR}^{71}$, os sulfonatos de lítio $\mathrm{SO}_{3} \mathrm{Li}^{72}$, sulfóxidos $\mathrm{SOR}^{73,74}$, as sulfonas $\mathrm{SO}_{2} \mathrm{R}^{75}$, os sulfonatos $\mathrm{SO}_{3} \mathrm{R}^{75}$, as sulfonamidas mono $\mathrm{SO}_{2} \mathrm{NHR}^{76}$ e dissubstituídas $\mathrm{SO}_{2} \mathrm{NR}_{2}{ }^{77}$.

$\mathrm{Na}$ série das piridinas, os grupos $\mathrm{SO}_{2} \mathrm{NR}_{2}^{78,79}$ e $\mathrm{SOR}^{80}$ foram amplamente utilizados em reações de ortometalação regiosseletivas. Estudos detalhados de Quéguiner e colaboradores demonstraram que a piperidinossulfonamida foi, sem dúvida, um dos melhores grupos ortodiretores contendo enxofre na realização de reações de ortometalação em sistemas piridínicos. Além disto, estes autores observaram que um excesso de LDA (2-3 equivalentes) é necessário para se obter compostos ortossubstituídos com bons rendimentos (Esquema 38) (99,80. $^{2}$.

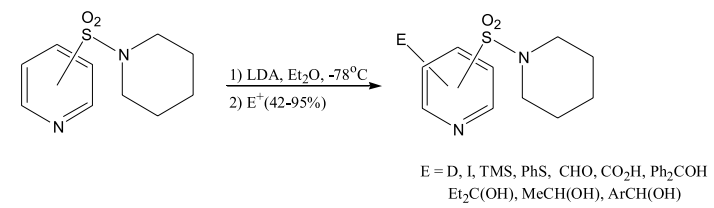

Esquema 38. Reações de ortometalação utilizando piperidinossulfonamida como grupo ortodiretor

O grupo fenilssulfóxido se revelou também interessante, na presença de LDA em THF a $-78^{\circ} \mathrm{C}$, já que a litiação regiosseletiva do anel piridínico pode ser realizada. Após adição dos respectivos eletrófilos, os compostos ortossubstituídos podem ser isolados com bons rendimentos (Esquema 39) ${ }^{81}$.

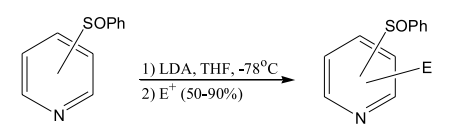

$\mathrm{E}=\mathrm{TMS}, \mathrm{PhS}, \mathrm{PhCH}(\mathrm{OH}), \mathrm{PhCMe}(\mathrm{OH})$

Esquema 39. Reações de ortometalação utillizando-se fenilssulfóxido como grupo ortodiretor.
O interesse do grupo ortodiretor do tipo fenilssulfóxido reside também na sua reatividade. Pode-se assim, obter-se rapidamente bipiridinas funcionalizadas substituindo-se diretamente o grupo fenilssulfóxido, empregando-se brometos de 2, 3 ou 4piridinilmagnésio (Esquema 40).

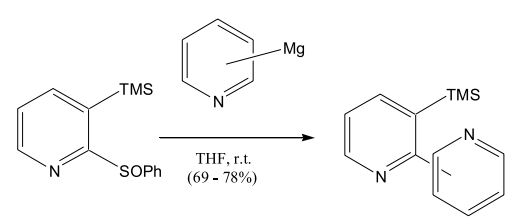

Esquema 40. Obtenção de bipiridinas funcionalizadas.

\section{CONCLUSÕES}

A reação de ortolitiação de compostos aromáticos e heteroaromáticos constitui um poderoso método sintético para a construção regiosseletiva de compostos orgânicos, utilizando-se condições reacionais brandas e com rendimentos elevados. Esta metodologia é claramente superior aos métodos sintéticos clássicos que empregam normalmente condições reacionais drásticas e que normalmente apresentam problemas de regiosseletividade, como por exemplo a substituição eletrofílica aromática.

\section{AGRADECIMENTOS}

Os autores agradecem pelas bolsas: de produtividade em pesquisa do CNPq para MVA e de recém-doutor da FAPEMIG para MVNS.

\section{REFERÊNCIAS}

1. Gilman, H.; Bebb, R.; J. Am. Chem. Soc. 1939, 61, 109.

2. Wittig, G.; Fuhrman, G.; Chem. Ber. 1940, 73, 1197.

3. a) Snieckus, V.; Chem. Rev. 1990, 90, 879; b) Snieckus, V.; Chem. Synthesis 1996, 191.

4. Quéguiner, G.; Marsais, F.; Snieckus, V.; Epsztajn, J.; Adv. Heterocycl. Chem. 1991, 52, 187.

5. Conley, R. A.; Barton, D. L.; Ortho Pharm. Corp., Chem. Abstr. 1998, 107, $11536 u$.

6. Hlasta, D. J.; Court, J. J.; Desai, R. C.; Tetrahedron Lett. 1991, 32, 7179.

7. Gschwend, H. W.; Rodriguez, H. R.; Org. React. 1979, 26, 1.

8. Shirley, D. A., Haarmont, T. E., Chun-Fong, C., J. Organomet. Chem. 1974, 69, 327.

9. Beak, P.; Meyers, A. I.; Acc. Chem. Res. 1986, 19, 356.

10. a) Chambers, R. D.; Drakesmith, F.G.; Musgrave, K. R.; J. Chem. Soc. 1965, 5045; b) Chambers, R. D.; Heaton, C. A.; Musgrave, K. R.; J. Chem. Soc. 1969, 1700 .

11. Abramovich, R.A; Saha, M.; Smith, E. M.; Coutts, R. T.; J. Am. Chem. Soc. 1967, 89. 1537.

12. Mallet, M.; Marsais, F.; Quéguiner, G.; Pastour, P.; C. R. Heb. Séances Acad. Sci. Ser. C. 1972, 275, 1439.

13. Mallet, M.; Marsais, F.; Quéguiner, G.; Pastour, P.; C. R. Heb. Séances Acad. Sci. Ser. C 1972, 275, 1535.

14. Ager, E.; Chivers, G. E.; Suschitzky, H.; J. Chem. Soc. Chem. Commun. 1972, 505.

15. Foulger, N. J.; Wakefield, B. J.; J. Organomet. Chem. 1974, 69, 161.

16. Gribble, G. W.; Saulmier, M. G.; Tetrahedron Lett. 1980, 21, 4137.

17. Gribble, G. W.; Saulmier, M. G.; Heterocycles 1993, 35, 151.

18. Marsais, F.; Granger, P.; Quéguiner, G.; J. Org. Chem. 1981, 46, 4494.

19. Gungor, T.; Marsais, F.; Quéguiner, G.; J. Organomet. Chem. 1981, 215, 139.

20. Kauffmann, T.; Wirthwein, R.; Angew. Chem. Int. Ed. Engl. 1971, 10, 20.

21. Marsais, F.; Laperdrix, B.; Gungor, T.; Mallet, M.; Quéguiner, G; J. Chem. Res. (S) 1982, 278.

22. Mallet, M.; Quéguiner, G.; Tetrahedron 1982, 38, 3035.

23. Mallet, M.; Quéguiner, G.; Tetrahedron 1985, 41, 3433.

24. Mallet, M.; Quéguiner, G.; Tetrahedron 1986, 42, 2253. 
25. Bunnett, J. F.; Victor, R. R.; J. Am. Chem. Soc. 1968, 90, 810;

26. Mallet, M.; Quéguiner, G.; Tetrahedron 1979, 35, 1625.

27. Bunnett, J. F.; Acc. Chem. Res. 1972, 55, 139.

28. Marsais, F.; Le Nard, G.; Quéguiner, G.; Synthesis 1982, 235.

29. Dewar, M. J. S.; Yamaguchi, Y.; Doraiswamy, S.; Sharma, S. D.; Suck, S. H.; Chem. Phys. 1979, 41, 21

30. Rocca, P.; Cochennec, C.; Marsais, F.; Thomas-dit-Dumont, L.; Mallet, M.; Godard, A.; Quéguiner, G.; J. Org. Chem. 1993, 58, 7832.

31. Clarke, A. J.; McNamara, S.; Meth-Cohn, O.; Tetrahedron Lett. 1974, 2373.

32. Kauffmann, T.; Angew. Chem. Int. Ed. Engl. 1979, 18, 1.

33. Marsais, F.; Quéguiner, G.; Tetrahedron 1983, 39, 2009.

34. Powers, J.C.; Ponticello, I.; J. Am. Chem. Soc. 1968, 90, 7102.

35. Kessar, S. V.; Gupta, Y. P.; Pahwa, P. S.; Singh, P.; Tetrahedron Lett. 1976, $36,3207$.

36. Lalezari, I.; Nabahi, S.; J. Heterocycl. Chem. 1980, 17, 1761.

37. Rocca, P.; Marsais, F.; Godard, A.; Quéguiner, G.; Tetrahedron 1993, 49, 49.

38. Miyaura, N.; Yanagi, T.; Suzuki, A.; Synth. Commun. 1981, 11, 513.

39. Tamura, Y.; Fujita, M.; Chen, L. C.; Ionue, M.; Kita, Y.; J. Org. Chem. 1981, $46,3564$.

40. Turner, J. A.; J. Org. Chem. 1983, 48, 3401.

41. Estel, L.; Linard, F.; Marsais, F.; Godard, A.; Quéguiner, G.; J. Heterocycl. Chem. 1989, 26, 105.

42. Smith, K.; Lindsay, C. M.; Morris, I. K.; Chem. Ind. 1988, 302.

43. Christensen, H.; Synth. Commun. 1975, 5, 65.

44. Ronald, R. C.; Winkle, M. R.; Tetrahedron 1983, 39, 2031.

45. Sibi, M. P.; Snieckus, V.; J. Org. Chem. 1983, 48, 1935.

46. Hasseberg, H. A.; Gerlach, H.; Helv. Chim. Acta 1988, 71, 957.

47. Winkle, M. R.; Ronald, R. C.; J. Org. Chem. 1982, 47, 2101.

48. Wada, A.; Kagatomo, S.; Nagai, S.; Chem. Pharm. Bull. 1985, 33, 1016.

49. Sengupta, S.; Snieckus, V.; Tetrahedron Lett. 1990, 31, 4267.

50. Miah, M. A. J.; Snieckus, V.; J. Org. Chem. 1985, 50, 5436.

51. Marsais, F.; Thesis, Université de Rouen, France 1984

52. Trécourt, F.; Mallet, M.; Marsais, F.; Quéguiner, G.; J. Org. Chem. 1988, 53, 1367.

53. Trécourt, F.; Gervais, B.; Mallet, M.; Godard, A.; Quéguiner, G.; J. Org. Chem. 1996, 61, 1673

54. Funk, A.; Divekar, P. V.; Can. J. Microbiol. 1959, 5, 317.
55. McInnes, A. G.; Smith, D.G.; Wright, J. L. C.; Vining, L. C.; Can. J. Chem. 1977, 55, 4159 .

56. Ferles, M.; Silhanka, A.; Collect. Czech. Chem. Commun. 1979, 44, 3137. 57. Beak, P.; Snieckus, V.; Acc. Chem. Res. 1982, 306, 15.

58. Meyers, A. I.; Gabel, R. A.; Tetrahedron Lett. 1978, 227.

59. Katritzky, A. R.; Rahimi-Rastgoo, J.; Ponkshe, N. R.; Synthesis 1981, 127.

60. a) Epsztajn, J.; Bieniek, A.; Brzezinski, J. Z.; Jozwiak, A.; Tetrahedron Lett. 1983, 24, 4735; b) De Souza, M.; Thesis, Université D’Orsay 1999; c) De Souza, M.; Dodd, R.H.; Heterocycles 1998, 2, 47, 811.

61. Watanabe, M.; Snieckus, V.; J. Am. Chem. Soc. 1980, 102, 1457.

62. Epsztajn, J.; Berski, Z.; Brzezinski, J. Z.; Jozwiak, A.; Tetrahedron Lett. 1980, 21, 4739 .

63. Meyers, A. I.; Gabel, R. A.; J. Org. Chem. 1982, 47, 2633.

64. Epsztajn, J.; Bieniek, A.; Plotka, M. W.; J. Chem. Res (S) 1986, 20

65. Epsztajn, J.; Bieniek, A.; Plotka, M. W.; J. Chem. Res (S) 1986, 18

66. Taylor, D. A.; Baradarani, M. M.; Martinez, S. J.; Joule, J. A.; J. Chem. Res (M) 1979, 4801.

67. Kelly, T. R.; Echavarren, A.; Chandrakumar, N. S.; Koksal, Y.; Tetrahedron Lett. 1984, 25, 2127.

68. Powell, R. G.; Smith, C. R.; Weisleder, D.; Muthard, D. A.; Clardy, J.; J. Am. Chem. Soc. 1979, 101, 2784.

69. Powell, R. G.; Smith, C. R.; Madrigal, R. V.; Planta Med. 1976, 30, 1.

70. Iwao, M.; Kuraishi, T.; Tetrahedron Lett. 1983, 24, 2649.

71. Horner, L.; Lawson, A. J.; Simons, G.; Phosphorus Sulfur 1983, 12, 779.

72. Figuly, G. D.; Martin, J. C.; J. Org. Chem. 1980, 45, 3728.

73. Furukawa, N.; Takahashi, F.; Kawai, T.; Kishimoto, K.; Ogawa, S.; Oae, S.; Phosphorus Sulfur 1983, 16, 167.

74. Iwao, M.; Lihama, T.; Mahalanabis, M.M.; Perier, H.; Snieckus, V.; J. Org. Chem. 1989, 54, 24.

75. Bonfiglio, J. N.; J. Org. Chem. 1986 51, 2833.

76. Lombardino, J. G.; J. Org. Chem. 1971, 36, 1843.

77. Watanabe, H.; Schwarz, R. A.; Hauser, C. R.; Lewis, J.; Slocum, D. W.; Can J. Chem. 1969, 47, 1543

78. Breant, P.; Marsais, F.; Quéguiner, G.; Synthesis 1983, 822.

79. Marsais, F.; Cronnier, A.; Trécourt, F.; Quéguiner, G.; J. Org. Chem. 1987, 52, 1133.

80. Furukawa, N.; Shibutani, T.; Fujihara, H.; Tetrahedron Lett. 1989, 30, 7091.

81. Perrier, H.; Thesis, Université du Canada 1989. 